Daniela Fraguas Schenkel $^{1}$
Jessica Dalék ${ }^{2}$

Vicente SPERB ANTONELLO²

\section{Artigo Original}

Palavras-chave Complicações infecciosas na gravidez Resistência microbiana a medicamentos

Urinálise

Urina/microbiologia

Keywords

Urinary tract infection/drug therapy Pregnancy complications, infectious

Drug resistance, microbial Urinalysis

Urine/microbiology

\title{
Prevalência de uropatógenos e sensibilidade antimicrobiana em uroculturas de gestantes do Sul do Brasil
}

\author{
Microbial etiology and susceptibility of community urinary tract \\ infections during pregnancy in the south of Brazil
}

\section{Resumo}

OBJETIVO: $\bigcirc$ objetivo do estudo foi avaliar a prevalência de germes e do perfil de sensibilidade a antimicrobianos a partir de uroculturas de pacientes gestantes atendidas em um hospital materno-infantil em Porto Alegre, Brasil. MÉTODOS: Foi realizado estudo transversal, retrospectivo e descritivo, no Hospital Fêmina, referência para atendimento às mulheres durante o pré-natal, parto e puerpério na cidade de Porto Alegre, Brasil. A partir de 1.558 uroculturas positivas, foram avaliados 482 resultados microbiológicos comunitários com perfil de sensibilidade antimicrobiana de uroculturas de gestantes em todas as idades gestacionais, atendidas no período de janeiro de 2007 a julho de 2013. RESULTADOS: $O$ padrão de sensibilidade apresentado nesta pesquisa mostra que a escolha para o tratamento de infecção urinária durante a gravidez deve ser nitrofurantoína (para infecções não complicadas) ou cefalosporinas de segunda geração, como cefuroxima (para infecções não complicadas e complicadas), evitando o uso de ampicilina, cefalosporinas de primeira geração e sulfametoxazol/trimetoprim. CONCLUSÃO: $\bigcirc$ tratamento empírico de infecção urinária na gravidez deve ser iniciado de acordo com os padrões de suscetibilidade descritos na literatura, e revisto após os resultados de cultura de urina.

\section{Abstract}

PURPOSE: Urinary tract infection (UTI) is one of the most common conditions during pregnancy. The aim of this study was to assess the prevalence of germs and the antimicrobial susceptibility profile in urine culture isolates from pregnant patients treated at a tertiary maternity hospital in Porto Alegre, Brazil. METHODS: A cross-sectional, retrospective and descriptive study was carried out at Hospital Fêmina, a leading institution in prenatal, birth and postnatal healthcare in the city of Porto Alegre, Brazil. A total of 482 microbial community results were analyzed out of 1,558 positive urine cultures of pregnant women in all gestational ages treated at Fêmina Hospital between January 2007 and July 2013. RESULTS: The susceptibility pattern presented in this research shows that the choice for UTI treatment during pregnancy should be nitrofurantoin (for uncomplicated infections) or second-generation cephalosporins such as cefuroxime (for uncomplicated and complicated infections), over ampicillin, first-generation cephalosporins and sulfamethoxazole/ trimethoprim. CONCLUSION: Empirical treatment for UTI in pregnancy should be started according to the susceptibility patterns described in the literature and re-analyzed after the results of the urine culture.

Correspondêncio

Vicente Sperb Antonello Serviço de Controle de Infecç̄ōo, Hospital Fêmin Rua Mostardeiro, 17 (EP: 91430-001 Porto Alegre (RS), Brasil

Recebido $21 / 01 / 2014$

Aceito com modificacōes

$03 / 02 / 2014$
Hospital Fêmina - Porto Alegre (RS), Brasil.

'Departamento de Ginecologia e Obstetrícia, Hospital Fêmina - Porto Alegre (RS), Brasil.

2Departamento de Prevenção e Controle de Infecções, Hospital Fêmina - Porto Alegre (RS), Brasil. 
Introdução

A infecção do trato urinário (ITU) é um problema freqüente na gestação, acometendo de 10 a $12 \%$ das gestantes $^{1-3}$. A ITU é definida como a invasão microbiana do trato urinário, desde a uretra até os rins. É classificada como cistite, pielonefrite e bacteriúria assintomática $(\mathrm{BA})^{4,5}$.

As mudanças anatômicas, fisiológicas e hormonais da gestação favorecem a ocorrência de infecções do trato urinário, ou a transformação da bacteriúria assintomática em ITU sintomática ${ }^{6,7}$. Estas alterações vão desde a dilatação do sistema coletor, aumento do tamanho renal, modificação da posição da bexiga, aumento do débito urinário, redução do tônus vesical com conseqüente aumento da capacidade vesical, além de alterações no pH urinário (urina mais alcalina) e diminuição da capacidade renal de concentrar a urina, excretando menores quantidades de potássio e maiores de glicose e aminoácidos. Todos esses fatores contribuem para a estase urinária e proliferação bacteriana no trato urinário, resultando muitas vezes em infecções urinárias na gestação ${ }^{7-10}$.

A Escherichia coli é a bactéria mais comumente encontrada nas infecções do trato urinário, tanto sintomáticas quanto assintomáticas, representando $70-80 \%$ dos casos. Outras bactérias gram-negativas (Klebsiella pneumoniae, Proteus mirabilis e bactérias do gênero Enterobacter), grampositivas (Staphylococcus saprophyticus, Streptococcus agalactiae e Enterococcus sp) e variavelmente fungos (Candida sp) são responsáveis pela restante dos $\operatorname{casos}^{3,7,11-14}$.

O diagnóstico precoce e o tratamento adequado devem ser prontamente implantados para evitar complicações maternas e fetais ${ }^{7}$. O conhecimento do microrganismo causador da infecção, suas características epidemiológicas e sua sensibilidade aos antimicrobianos é de fundamental importância, a fim de proporcionar maior eficácia do tratamento e impedir complicações às gestantes ${ }^{15}$.

Este estudo tem por objetivo analisar a prevalência de germes e o perfil de sensibilidade antimicrobiana em isolados de uroculturas de pacientes gestantes atendidas em hospital terciário materno-infantil no Município de Porto Alegre. Esta avaliação tem extrema relevância, visto o crescente surgimento de resistência bacteriana e o limitado arsenal antimicrobiano para uso seguro na gestação.

\section{Métodos}

Foi realizado estudo transversal, retrospectivo e descritivo no Hospital Fêmina, instituição referência para o atendimento a mulheres durante o pré-natal, parto e puerpério na cidade de Porto Alegre, Rio Grande do Sul, Brasil. A partir de 1.558 uroculturas positivas, foram avaliados 482 resultados microbiológicos comunitários com perfil de sensibilidade antimicrobiana de uroculturas de gestantes em todas as idades gestacionais, atendidas no Hospital Fêmina no período de janeiro de 2007 a julho de 2013. As uroculturas foram identificadas a partir da comunicação regular do Laboratório de Microbiologia da instituição sobre os resultados microbiológicos ao Serviço de Controle de Infecção Hospitalar do Hospital Fêmina. Adicionalmente, foram avaliadas informações demográficas das pacientes, idade gestacional e o quadro clínico (bacteriúria assintomática, cistite ou pielonefrite), informações estas contidas nas fichas de notificação do Serviço de Controle de Infecção Hospitalar do Hospital Fêmina. O critério de inclusão para o estudo é a urocultura da comunidade de gestante atendida no Hospital Fêmina. A definição de urocultura da comunidade utilizada foi urocultura coletada até 72 horas do início da internação, conforme recomendação do $\mathrm{CDC}^{16}$. Foram incluídas apenas pacientes sem internação recente no Hospital Fêmina.

O crescimento bacteriano significativo (urocultura positiva) foi considerado a partir de $10^{5}$ unidades formadoras de colônias de bactérias obtidas a partir de um mililitro de urina não centrifugada ${ }^{17}$. Foram realizados testes de sensibilidade dos isolados bacterianos aos antibióticos comumente utilizados (amicacina, gentamicina, ampicilina, cefalotina, cefoxitina, cefepima, ceftazidima, piperacilina/tazobactam, ertapenem, nitrofurantoína, norfloxacino, ciprofloxacino e sulfametoxazol-trimetoprim).

Para a análise estatística, foram utilizados avaliação descritiva com média e desvio padrão para variáveis contínuas e freqüência e percentagem para variáveis categóricas. Na comparação para as médias entre grupos, divididos a cada ano para prevalência de germes e perfil de sensibilidade aos antimicrobianos, utilizamos o teste $t$ de Student e, para análise das frequências, o teste do $\chi^{2}$. Para avaliar o risco comparativo de desenvolvimento de ISC entre os grupos de parto cesáreo e vaginal, utilizamos risco relativo (RR) e intervalo de confiança (IC) de 95\%. Foi utilizado como banco de dados o programa Microsoft Excel e, para análise, o programa SPSS (Statistical Package for Social Sciences) para Windows.

O estudo foi aprovado pelo Comitê em Ética em Pesquisa do Grupo Hospitalar Conceição, em 10 de novembro de 2013, com o número de registro 22647313.0.0000.5530. 
Resultados

No período de janeiro de 2007 até julho de 2013, 481 resultados de uroculturas de 454 pacientes gestantes com idades entre 12 e 44 anos (média de 24,2 anos e desvio padrão de $\pm 6,6$ ), atendidas no Hospital Fêmina, foram incluídas no presente estudo. Em 427 pacientes (94,0\%), a infecção era monomicrobiana e, em 27 pacientes, a urocultura demonstrava crescimento de dois ou mais germes $(5.9 \%)$.

Entre os organismos isolados, E. coli foi o germe predominante (363/481; 75,4\%), seguido por Enterococcus $s p(34 / 481 ; 7.0 \%)$, Streptococcus agalactiae $(19 / 481 ; 3,9 \%)$, P. mirabilis (18/481; 3,7\%) e K. pneumoniae (16/481;3,3\%). Outras enterobactérias corresponderam a 2,0\% dos casos (10/481). Todas as outras espécies representaram menos de $5 \%$ dos isolados $(21 / 481 ; 4,3 \%)$.

Em relação ao padrão de suscetibilidade para E. coli, emergem como as drogas seguras na gestação testadas mais ativas as cefalosporinas de quarta, terceira e segunda geração, Piperacilina/Tazobactam e Nitrofurantoína, conforme a Tabela 1. Quando avaliados os outros germes prevalentes, Enterococcus sp e Streptococcus agalactiae demonstraram boa sensibilidade para os antibióticos testados [Ampicilina (93\%) e Vancomicina (100\%)], assim como Protens mirabilis e Klebsiella pneumoniae, que apresentaram perfil de sensibilidade semelhante ao encontrado para E. coli (Tabela 2).

Em 394 uroculturas de pacientes, foi possível identificar o período gestacional em que a paciente se encontrava. A presença de E. coli em relação ao total de germes isolados foi mais prevalente no primeiro (84\%) e segundo trimestres (84\%), comparado ao terceiro trimestre $(63,7 \%)$, embora não tenha havido diferença significativa entre os grupos. Quando observado o padrão de sensibilidade a E. coli entre os grupos, não houve diferença para nenhum dos antibióticos testados, sendo o perfil de suscetibilidade muito semelhante ao grupo total de pacientes.

As uroculturas foram divididas conforme síndrome clínica apresentada (bacteriúria assintomática, cistite e pielonefrite). Esta informação estava disponível em 471 casos. Para bacteriúria assintomática, E. coli correspondeu a $64 \%(132 / 205)$ dos casos; para cistite, $80,7 \%(67 / 83)$ e para pielonefrite, $84,7 \%(155 / 183)$. Novamente, não houve diferença entre os grupos para padrão de suscetibilidade aos antimicrobianos testados.

Foi analisada a evolução do padrão de suscetibilidade em isolados de uroculturas aos antibióticos testados de 2010 a 2013 (Figura 1). É possível notar uma tendência positiva no padrão de suscetibilidade para Ampicilina e Sulfametoxazol/Trimetoprim. Os demais antibióticos não demonstram modificações relevantes ao longo dos anos avaliados no presente estudo.
Tabela 1. Padrão de suscetibilidade das amostras de Escherichia colia partir dos isolados de urocultura de gestantes

\begin{tabular}{|c|c|c|c|c|c|c|}
\hline \multirow{3}{*}{$\begin{array}{l}\text { Agente antimicrobiano } \\
\text { Ampicilina }\end{array}$} & \multicolumn{6}{|c|}{ Suscetibilidade [\%,(número de isolados)] } \\
\hline & \multicolumn{2}{|c|}{ S } & \multicolumn{2}{|c|}{ I } & \multicolumn{2}{|c|}{$\mathbf{R}$} \\
\hline & 54,6 & 166 & 0,05 & 2 & 44,9 & 137 \\
\hline Cefalosporina de $1^{\circ}$ geração & 71,8 & 247 & 18,7 & 64 & 9,5 & 33 \\
\hline Cefalosporina de 2 • geração & 97,5 & 272 & 1,4 & 4 & 1,1 & 3 \\
\hline Cefalosporina de 3 • geração & 98,3 & 290 & 0 & 1,7 & 5 & \\
\hline Cefalosporina de 4 geração & 98,4 & 325 & 0 & 1,6 & 5 & \\
\hline Ciprofloxacino & 96,1 & 272 & 0 & 3,9 & 11 & \\
\hline Colistina & 100 & 249 & 0 & 0 & & \\
\hline Ertapenem & 100 & 302 & 0 & 0 & & \\
\hline Gentamicina & 96,9 & 286 & 0 & 3,1 & 9 & \\
\hline Nitrofurantoina & 92,1 & 257 & 5 & 14 & 2,9 & 8 \\
\hline Piperacilina/Tazobactam & 98,1 & 206 & 0,4 & 1 & 1,5 & 3 \\
\hline Sulfametoxazol/Trimetoprim & 63,6 & 165 & 0,3 & 1 & 36,1 & 93 \\
\hline Tigeciclina & 100 & 100 & 0 & 0 & & \\
\hline
\end{tabular}

S: Suscetível; I: Intermediário; R: Resistente.

Tabela 2. Padrão de suscetibilidade de Proteus mirabilis e Klebsiella pneumoniae em isolados de urocultura de gestantes

\begin{tabular}{|c|c|c|c|c|}
\hline \multirow{3}{*}{ Agente antimicrobiano } & \multicolumn{4}{|c|}{ Suscetibilidade (\% dos casos) } \\
\hline & \multicolumn{2}{|c|}{$\begin{array}{l}\text { Klebsiella pneumoniae } \\
\text { (16 casos) }\end{array}$} & \multicolumn{2}{|c|}{$\begin{array}{l}\text { Proteus mirabilis } \\
\text { (18 casos) }\end{array}$} \\
\hline & $s$ & $\mathbf{R}$ & $s$ & $\mathbf{R}$ \\
\hline Ampicilina & 28,6 & 71,4 & 94 & 6 \\
\hline Cefalosporina de $1{ }^{\circ}$ geração & 81,3 & 28,7 & 94 & 6 \\
\hline Cefalosporina de $2{ }^{\circ}$ geração & 100 & 0 & 100 & 0 \\
\hline Cefalosporina de $3{ }^{\circ}$ geração & 100 & 0 & 100 & 0 \\
\hline Cefalosporina de $4^{\circ}$ geração & 100 & 0 & 100 & 0 \\
\hline Ertapenem & 100 & 0 & 100 & 0 \\
\hline Colistina & 100 & 0 & 0 & 100 \\
\hline Ciprofloxacino & 100 & 0 & 100 & 0 \\
\hline Gentamicina & 100 & 0 & 100 & 0 \\
\hline Nitrofurantoína & 69 & 31 & 0 & 100 \\
\hline Piperacilina/Tazobactam & 83 & 17 & 100 & 0 \\
\hline Sulfametoxazol/Trimetoprim & 100 & 0 & & 91 \\
\hline Tigeciclina & & 100 & 0 & \\
\hline
\end{tabular}

S: Suscetível; R: Resistente.

\section{Discussão}

Infecção do trato urinário é uma das condições infecciosas mais comuns na gestação. Este estudo analisou a epidemiologia e padrão de suscetibilidade dos uropatógenos mais prevalentes aos antibióticos rotineiramente utilizados no tratamento de bacteriúria assintomática, cistite e pielonefrites.

De acordo com o descrito na literatura ${ }^{11-15,17-19}$, E. coli foi o germe mais prevalente, seguido de Enterococcus sp, Streptococcus agalactiae e outras enterobactérias como 


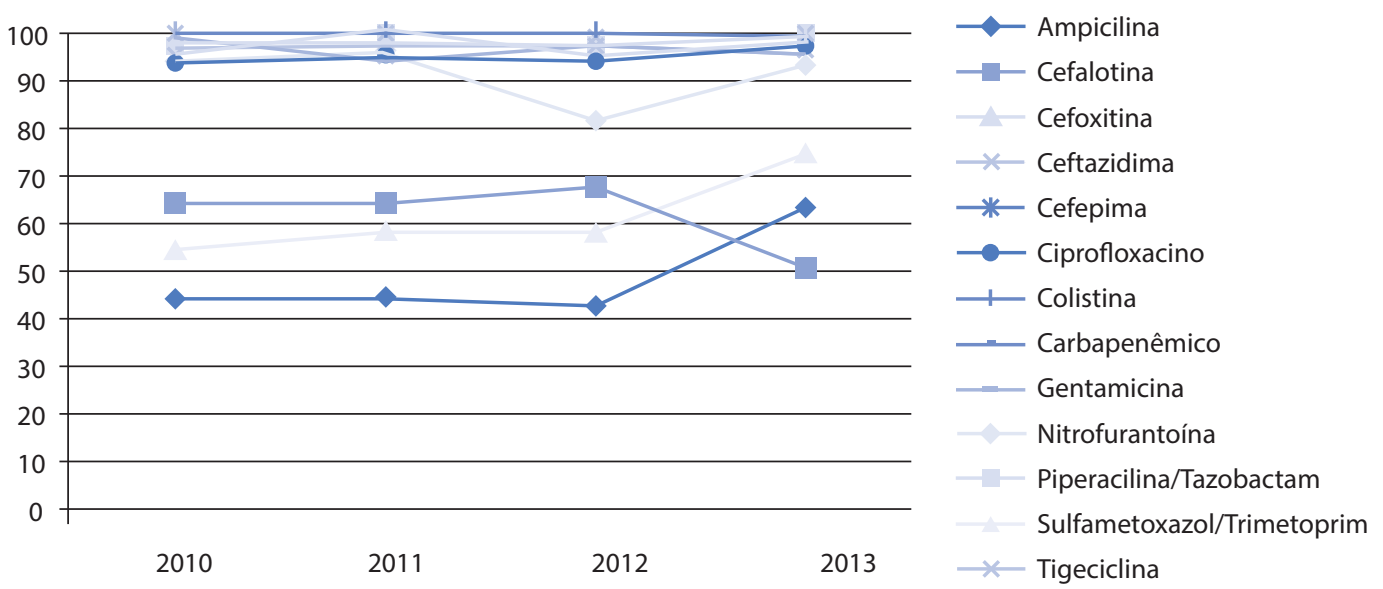

Figura 1. Comparação do padrão de suscetibilidade aos antibióticos testados em isolados de uroculturas de 2010 a 2013.

P. mirabilis e K. pneumoniae. Esta situação pode ser explicada pela patogenicidade inerente para colonização urinária e as características de adesão do E. coli no trato urinário. Adicionalmente, o E. coli faz parte da flora normal do trato gastrointestinal, e é a principal fonte de agente infeccioso para bacteriúria assintomática em mães no pré-natal ${ }^{18,19}$. Neste estudo, dividindo as pacientes por idade gestacional e por síndrome clínica (bacteriúria assintomática, cistite e pielonefrite), não houve diferença na prevalência de germes, sendo novamente E. coli o germe mais comumente relacionado à ITU em gestantes. Esta última situação pode ser explicada pela evolução de colonização do trato urinário até a síndrome clínica, sendo, portanto, o mesmo germe implicado na ITU.

Os padrões de suscetibilidade, especialmente ao principal patógeno (E. coli), são bastante assemelhados com o descrito na literatura, ou seja, ampicilina e sulfametoxazol/trimetoprim foram os antimicrobianos menos ativos ${ }^{20,21}$. Adicionalmente, houve resistência frequente em gram-negativos para cefalosporinas de primeira geração. Esta situação está de acordo com a utilização ampla dessas medicações nos últimos anos para tratamento de ITU em gestantes e não-gestantes, acarretando aumento na resistência bacteriana ${ }^{21-25}$. Todos outros antibióticos mostraram ótimo padrão de suscetibilidade. Tendo em vista o restrito arsenal terapêutico para as gestantes, devido ao risco de mal-formações fetais, cefuroxima e nitrofurantoína emergem como excelentes opções no tratamento das síndromes urinárias infecciosas comunitárias em gestantes ${ }^{20}$.

O presente estudo avaliou a evolução do padrão de suscetibilidade de 2010 até 2013. É possível observar que não houve modificações para a maioria dos grupos estudados. No entanto, é preciso discutir três antimicrobianos em especial. Cefalosporinas de primeira geração têm sido amplamente utilizadas e recomendadas no tratamento empírico de infecções urinárias ${ }^{26}$. Dessa maneira, a diminuição no padrão de suscetibilidade é esperada, como mostra a Figura 1. Por outro lado, nos últimos anos, tanto ampicilina quanto sulfametoxazol/trimetoprim têm sido evitados

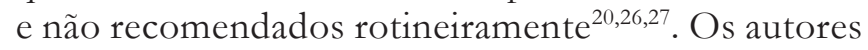
acreditam que esta prática tem resultados na diminuição da prescrição, utilização e consequentemente da pressão de resistência, especialmente de gram-negativos, sobre ampicilina (aumento na suscetibilidade de 45 para $64 \%$ ) e sulfametoxazol/trimetoprim (aumento de 59 para 75\% no padrão de suscetibilidade).

Em relação às limitações do estudo, o pequeno número de isolados de urocultura deve ser considerado. Adicionalmente, a limitação de testagem a alguns antibióticos no período de 2007 a 2009 evitou a comparação de evolução no padrão de suscetibilidade aos antibióticos testados neste período.

Em conclusão, o padrão de suscetibilidade apresentado neste estudo revela que a escolha para tratamento de ITU na gestação deve ser nitrofurantoína (para infecções não-complicadas) ou cefalosporinas de primeira geração como Cefuroxima (para infecções não-complicadas e complicadas), em detrimento de ampicilina, cefalosporinas de primeira geração e sulfametoxazol/trimetoprim. É importante destacar o aumento de suscetibilidade para ampicilina e sulfametoxazol/trimetoprim desde 2010 até 2013. Estes dados devem ser avaliados com cautela. Novos estudos e o seguimento do mesmo mostrarão a tendência de suscetibilidade para estes e outros antibióticos na prática clínica. 
Finalmente, é fundamental que a terapêutica empírica nas síndromes infecciosas urinárias na gestação seja iniciada conforme os padrões de suscetibilidade descritos na literatura ${ }^{28}$, devendo ser avaliada imediatamente após o resultado da urocultura para o correto tratamento direcionado ao patógeno implicado.

\section{Referências}

1. Dotters-Katz SK, Heine RP, Grotegut CA. Medical and infectious complications associated with pyelonephritis among pregnant women at delivery. Infect Dis Obstet Gynecol. 2013;2013:124102.

2. Alós Jl. [Epidemiology and etiology of urinary tract infections in the community. Antimicrobial susceptibility of the main pathogens and clinical significance of resistance]. Enferm Infecc Microbiol Clin. 2005;23 Suppl 4:3-8. Spanish.

3. Le J, Briggs $G G$, McKeown A, Bustillo $G$. Urinary tract infections during pregnancy. Ann Pharmacother. 2004;38(10):1692-701.

4. Schnarr J, Smaill F. Asymptomatic bacteriuria and symptomatic urinary tract infections in pregnancy. Eur J Clin Invest. 2008;38 Suppl 2:50-7.

5. Nicolle LE. Asymptomatic bacteriuria: review and discussion of the IDSA guidelines. Int J Antimicrob Agents. 2006;28 Suppl 1:S42-8.

6. Heilberg IP, Schor N. Abordagem diagnóstica e terapêutica na infecção do trato urinário - ITU. Rev Assoc Med Bras. 2003;49(1):109-16.

7. van Brummen HJ, Bruinse HW, van der Bom JG, Heintz AP, van der Vaart $\mathrm{CH}$. How do the prevalences of urogenital symptoms change during pregnancy? Neurourol Urodyn. 2006;25(2):135-9.

8. Beydoun $\mathrm{SN}$. Morphologic changes in the renal tract in pregnancy. Clin Obstet Gynecol. 1985;28(2):249-56.

9. Duarte G, Marcolin AC, Gonçalves CV, Quintana SM, Berezowski AT, Nogueira AA, et al. [Urinary infection in pregnancy: analysis of diagnostic methods and treatment]. Rev Bras Ginecol Obstet. 2002;24(7):471-7. Portuguese.

10. Salcedo $M M$, El Beitune $P$, Salis MF, Jiménez $M F$, Ayub AC. Infecção urinária na gestação. RBM Rev Bras Med. 2010;67(8):270-3.

11. Lopes HV, Tavares W. Diagnóstico das infecções do trato urinário. Rev Assoc Med Bras. 2005;51 (6):306-8.

12. Orenstein R, Wong ES. Urinary tract infections in adults. Am Fam Physician. 1999;59(5):1225-34, 1237.

13. Dias Neto JA, Martins ACP, Silva LD, Tiraboschi RB, Domingos $\mathrm{AL}$, Cologna $\mathrm{A}$, et al. Community acquired urinary tract infection: etiology and bacterial susceptibility. Acta Cir Bras. 2003;18(5):33-5.

14. Mazor-Dray E, Levy A, Schlaeffer F, Sheiner E. Maternal urinary tract infection: is it independently associated with adverse pregnancy outcome? J Matern Fetal Neonatal Med. 2009;22(2): 124-8.

15. Wagenlehner FM, Pilatz A, Naber KG, Perletti G, Wagenlehner $C M$, Weidner W. Anti-infective treatment of bacterial urinary tract infections. Curr Med Chem. 2008;15(14):1412-27.
16. CDC/NHSN Surveillance Definitions for Specific Types of Infections [Internet]. Georgia: Centers for Disease Control and Prevention; 2014. [cited 2014 Jan 6]. Available at: <http://www.cdc.gov/ $\mathrm{nhsn/pdfs/pscmanual/17pscnosinfdef \_ current.pdf>.}$

17. Lucas $M$, Cunningham FG. Urinary infection in pregnancy. Clin Obstet Gynecol. 1993;36(4):855-68.

18. Nicolle LE, Bradley S, Colgan R, Rice JC, Schaeffer A, Hooton TM, et al. Infectious Diseases Society of America guidelines for the diagnosis and treatment of asymptomatic bacteriuria in adults. Clin Infect Dis. 2005;40(5):643-54.

19. Andabati G, Byamugisha J. Microbial aetiology and sensitivity of asymptomatic bacteriuria among ante-natal mothers in Mulago hospital, Uganda. Afr Health Sci. 2010;10(4):349-52.

20. Schito GC, Naber KG, Botto H, Palou J, Mazzei T, Gualco L, et al. The ARESC study: an international survey on the antimicrobial resistance of pathogens involved in uncomplicated urinary tract infections. Int J Antimicrob Agents. 2009;34(5):407-13.

21. Paula LG, Krahe C, Carvalho RL. Infecção urinária e gestação. Femina. 2005;33(3):209-13.

22. McNulty CA, Richards J, Livermore DM, Little P, Charlett A, Freeman $\mathrm{E}$, et al. Clinical relevance of laboratory-reported antibiotic resistance in acute uncomplicated urinary tract infection in primary care. J Antimicrob Chemother. 2006;58(5):1000-8.

23. Duarte G. Diagnóstico e condutas nas infecções ginecológicas e obstétricas. 2a ed. Ribeirão Preto: FUNPEC; 2004.

24. Jeyabalan A, Lain KY. Anatomic and functional changes of the upper urinary tract during pregnancy. Urol Clin North Am. 2007;34(1): 1-6.

25. Teichmann A, Agra HN, Nunes LS, Rocha MP, Renner JD, Possuelo $L G$, et al. Antibiotic resistance and detection of the sul2 gene in urinary isolates of Escherichia coli in patients from Brazil. J Infect Dev Ctries. 2014;8(1):39-43.

26. Pires MC, Frota KS, Martins Junior PO, Correia AF, Cortez-Escalante JJ, Silveira CA. Prevalência e suscetibilidades bacterianas das infecções comunitárias do trato urinário, em hospital universitário de Brasília, no período de 2001 a 2005. Rev Soc Bras Med Trop. 2007;40(6): 643-7.

27. Gupta K, Hooton TM, Naber KG, Wullt B, Colgan R, Miller LG, et al. International clinical practice guidelines for the treatment of acute uncomplicated cystitis and pyelonephritis in women: A 2010 update by the Infectious Diseases Society of America and the European Society for Microbiology and Infectious Diseases. Clin Infect Dis. 201 1;52(5):e 103-20.

28. Wirtz VJ, Dreser A, Gonzales R. Trends in antibiotic utilization in eight Latin American countries, 1997-2007. Rev Panam Salud Publica. 2010;27(3):219-25. 